\title{
Early Development of the Terrestrial Planets: A Comparative Study
}

\author{
by Valery L. Barsukov and Alexandr T. Basilevsky
}

\begin{abstract}
Using the results of Soviet work, this article summarizes current ideas on the early evolution of the inner planets and their satellites. Initial accretion of the proto-planetary material was followed around 4.5 Ga ago by its very early differentiation along broadly similar paths on the Earth, Moon, Mars, Mercury, Venus and some meteorites. Intense meteoroidal bombardment, perhaps lasting until $3.8 \mathrm{Ga}$, was accompanied by impact metamorphism, melting and degassing. Volatiles were lost from the smaller bodies but accumulated on the larger ones to form their atmospheres and hydrospheres. Basaltic volcanism outlasted these events, though its relationship to the older "terra" material is not understood.
\end{abstract}

\section{The Earth}

Earth is the largest body among the inner planets (Table 1). Its large mass has hindered the escape of volatiles, and this fact together with its relative proximity to the Sun has led to the formation of the atmosphere, hydrosphere and highly developed life. Under the ocean floors the Earth's crust is composed mainly of basalts beneath the continents granite gneisses overly a "basaltic" layer, though we have no direct evidence of this composition.

Vigorous endogenic and magmatic activity combines with intense exogenic processes to cause the marked cycling of rock material. The essence of this was expressed long ago in the concept of the geochemical cycle: weathering, transportation and deposition of sediments, their metamorphism and melting. This cycle appears to have operated in much the same way throughout that part of geological time that we can date adequately $(\simeq 3.8 \mathrm{Ga})$. For example, the isotopic difference between organic carbon and carbonates, which reflects the vitality of living organisms, proves to be almost identical for very ancient metasedimentary rocks and for recent sediments, indicating that, despite their evolutionary trends, the global geochemical role of organisms has not changed in this period of time. To obtain information about earlier stages in the Earth's evolution (back to $4.7 \mathrm{Ga}$ ) we

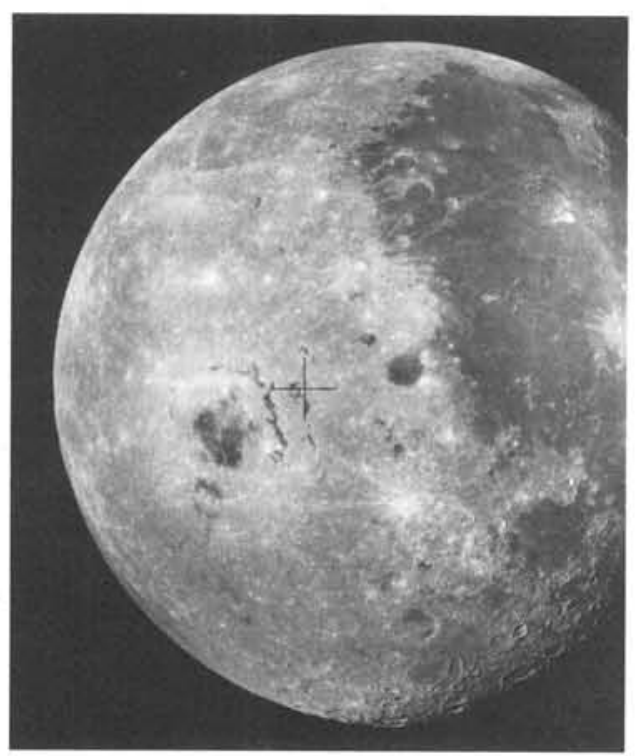

Figure 1: The Moon from Zond 8. To the right, dark basalt plains of the Oceanus Procellarum; to the left, relatively light lunar terrae. The circular feature to the left of the marking cross is the multi-ring impact Orientale Basin, 900 $\mathrm{km}$ across. have to turn to other planetary bodies not affected by the later intense geological activity common on Earth.

\section{The Moon}

The Moon is characterized by two major types of terrain: the so-called terrae or highlands and the maria (Fig. 1). Isotopic studies show that the differentiation of the lunar substance and the separation of a terra-type crust took place 4.5-4.6 Ga ago, almost simultaneously with the end of accretion of preplanetary substances into the lunar body.

Light-coloured terrae occupy about $84 \%$ of the lunar surface. Chemically and mineralogically, most of the terra rocks are similar to feldspathic igneous rocks of the terrestrial anorthosite-norite-troctolite (ANT) group, and they may represent a kind of planetological equivalent of Earth's continental crust. However, a specific geochemical feature of the lunar ANT rocks is their shortage of volatile elements and compounds - water and $\mathrm{CO}_{2}$ are absent, and alkalis are present only in extremely small quantities - which makes them very different from their terrestrial counterparts. This situation appears to be related to the small mass of the Moon, which was incapable of holding back volatiles released in the course of its initial planetary differentiation.

Terra rocks, at least to a depth of 10 to $20 \mathrm{~km}$, are breccias with substantial impact melt admixtures, which formed due to intensive meteoroidal bombardment. Study of lunar samples shows that the latter took place 3.9-4.6 Ga ago. Terra relief and elemental composition appear to have been greatly influenced by this bombardment; terra breccias, for example, have abundant "meteoroidal" siderophile elements.

The lunar maria, which occupy $16 \%$ of the Moon's surface, are volcanogenic plains composed of basaltic flows filling depressions in the terra relief. Lunar mare basalts are generally similar to terrestrial continental flood and ocean floor basalts, except again in their extremely low content of volatiles. Samples collected from the lunar maria have been dated in the range 3.1-3.9 Ga, but some terra breccia samples contain fragments of mare-type basalts whose age is approximately $4.25 \mathrm{Ga}$. Photogeological analysis of terra surfaces also provides evidence of these very early manifestations of basaltic volcanism.

Basaltic volcanism appears to have started during the period of intensive meteoroidal bombardment, with strong and relatively frequent impacts facilitating the process. This phenomenon may be regarded as a general process of differentiation of the interior of the planets. Studies of lunar samples and indirect dating of mare basalts using the density of superimposed impact craters indicate that this volcanism came slowly to an end 3-2 Ga ago. Since then meteorite impacts have been infrequent.

Comparing this chronology with that on the Earth, it seems possible that at least a portion of the terrestrial crust could 
also have separated at an early stage from the material that presently composes the mantle. Likewise we may assume that a similar meteoroidal bombardment of the Earth may have taken place 4.6-3.8 Ga ago, creating conditions under which both life and the geochemical cycle may have originated.

\section{Mercury}

Somewhat larger than the Moon but much smaller than the Earth (Table 1), Mercury has no atmosphere. In terms of landform and geology it bears a great resemblance to the Moon. Its surface is peppered with impact craters which are extensive on light-coloured highlands and fairly uncommon on dark flat plains. The morphology of the latter suggests that they, like the lunar maria, are areas of basaltic lava. Spectrophotometric and polarimetric data also indicate a general similarity with the lunar surface with respect to chemical and mineral composition. The most remarkable feature of Mercury is a system of elongate ridges, apparently of fault origin, that are commonly acknowledged to have been formed as a result of volume decrease during cooling.

\begin{tabular}{lcc} 
& Radius $(\mathrm{km})$ & $\begin{array}{c}\text { Gravity } \\
\left(\mathrm{cm} / \mathrm{sec}^{2}\right)\end{array}$ \\
\hline Earth & 6,378 (equatorial) & 978 \\
Moon & 1,738 & 162 \\
Mercury & 2,439 (equatorial) & 370 \\
Mars & 3,394 (equatorial) & 371 \\
Venus & $6,051.5$ (equatorial) & 890 \\
Phobos & $19 \times 21 \times 27$ (dimensions) & $<1$ \\
Deimos & $11 \times 12 \times 15$ (dimensions) & \\
& &
\end{tabular}

TABLE 1: Comparative sizes and gravity constants for inner planetary bodies

\section{Mars}

Mars is also characterized by two major types of terrain: ancient cratered highlands which are located mostly in the southern hemisphere and younger, apparently basaltic, maretype plains which are primarily concentrated in the northern hemisphere (Fig. 2). Gamma-ray spectroscopic data collected by the Mars 5 probe orbiting the planet suggest that the martian terrae may be similar to lunar ANT rocks in terms of uranium and thorium content. XRF measurements of soil at the Viking 1 and 2 landing sites indicate a mafic composition, reflecting perhaps the average composition of the upper portion of the martian crust.

There are also geological features on Mars that are more similar to those of Earth. Most important are a number of gigantic shield basaltic volcanoes, though these are much larger than their terrestrial counterparts, apparently because of the lower gravity on Mars (Table 1). There are also pronounced fault zones and a system of huge canyons on Mars, regarded by some as equivalent to terrestrial rift zones.

Since Mars has a relatively large mass and is located farther from the Sun than Earth, Moon and Mercury, it contains a certain amount of volatiles. There is an atmosphere on Mars which, although much more subtle than on Earth, facilitates the operation of eolian processes. As in the case of the Earth, the martian axis of rotation is inclined to the orbital plane, leading to annual changes of seasons best exemplified in its seasonal polar caps consisting of frozen $\mathrm{H}_{2} \mathrm{O}$ and $\mathrm{CO}_{2}$. Great amounts of $\mathrm{H}_{2} \mathrm{O}$-ice also appear to be buried in the uppermost portion of the martian crust, causing such phenomena as degradation of permafrost and the formation of ejecta mud flows around impact craters that penetrate icebearing horizons. In certain areas there are fluvial valleys
(Fig. 2) which, though dry at present, were active in previous geological epochs.

In simple terms, thus, two major types of crustal material can be distinguished: plain and shield volcano basalts and terra material which, despite its volatile content, is somewhat similar to lunar terra rocks of the ANT group. The martian terrae also exhibit evidence of intense meteoroidal impact, indicating that they formed at a very early stage. However, the geological history of Mars is more complex than that of the Moon and Mercury. In addition to marinetype volcanogenic plains superimposed on cratered highlands, Mars is characterized by later manifestations of active shield-type basaltic volcanism, intense tectonic movements as well as by the formation of fluvial-type valleys and other processes related to the presence of volatiles.

\section{The Satellites of Mars}

Mars has two satellites, Phobos and Deimos, which due to their extremely small dimensions are of great interest to comparative studies. They appear to be small asteroids captured by Mars, as indicated by their irregular nonspherical shapes (Table 1), and neither has an atmosphere. TV imagery shows that their terrain is of one type only, intensely cratered and geomorphologically comparable with the lunar terrae. However, in addition to impact craters, there is a set of continuous parallel grooves on Phobos, which appear to be fractures developed as a result of the formation of Stickney, its major crater.

The low average density of Phobos and Deimos, determined from orbital variations of the spacecraft, and their reflection spectra indicate that they are composed by primitive nondifferentiated material similar to that of carbonaceous chondrites $\mathrm{Cl}$ or $\mathrm{C} 2$. There is no indication that the Martian satellites were involved in planetary differentiation processes, and a shell structure is not present. Thus there appears to be a certain critical dimension for planetary bodies below which no differentiation of the original planetary substance took place.

\section{The Asteroid Belt}

Asteroids are of great significance for comparative planetology because they range from "geologically passive" minor bodies like Phobos and Deimos to planetary bodies with their own course of evolution. However, since asteroids have not as yet been studied from spacecraft, all progress in this field has been through ground-based telescopic observations.

Spectroscopic data permits a classification of asteroids according to their composition. It is now clear, for example, that there is a zoning in the distribution of different types according to their distance from the Sun. This zoning clearly indicates that the composition of the original protoplanetary material greatly varies even within the asteroidal belt, depending upon the distance from the Sun. Considerable

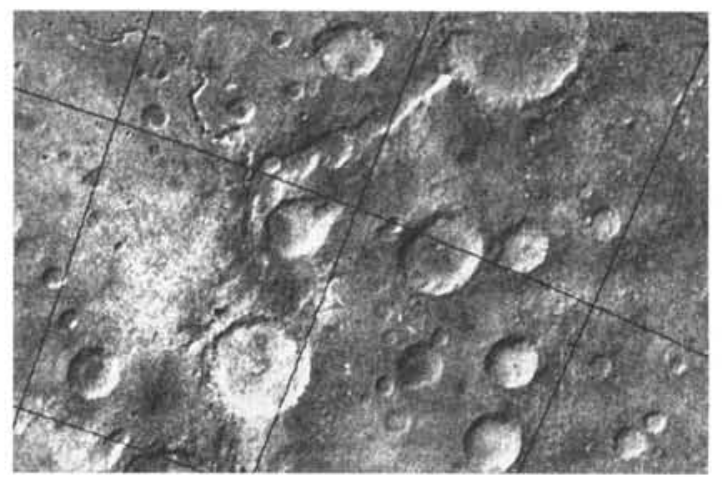

Figure 2: Terra area of Mars to the north of the Argyre Basin $(450 \times 600 \mathrm{~km})$. In addition to the craters typical of the terrae, the sinuous valleys of fluvial genesis are seen in the upper left corner. 


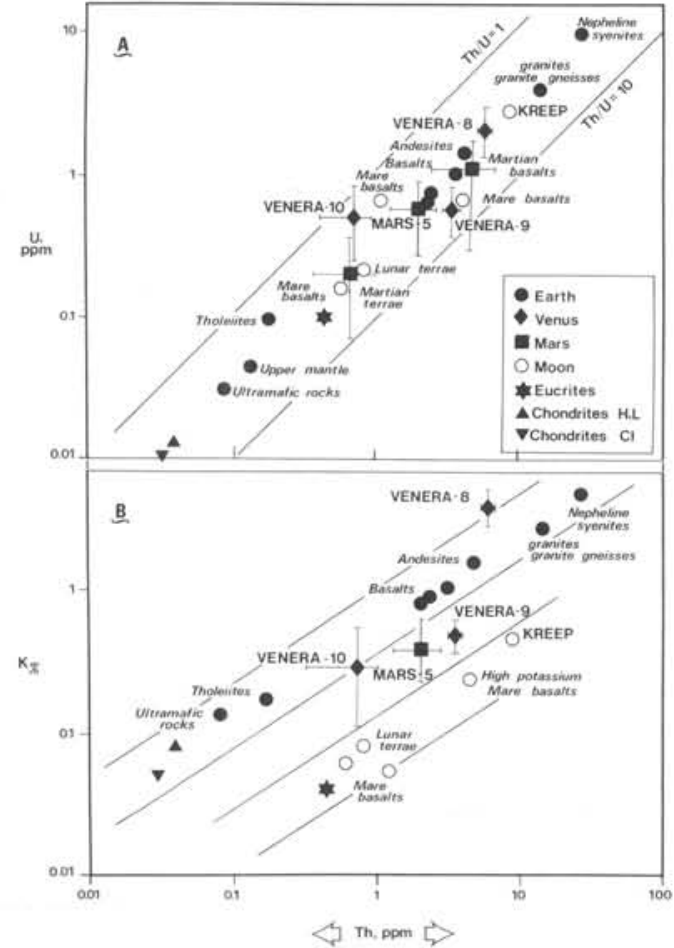

Figure 3: U-Th systematics (A) and $K$-Th systematics (B) for Earth, Venus, Mars, Moon and some meteorites.

discretion is therefore required when using different types of meteorites as prototypes for the original composition of the Earth and other planetary bodies.

\section{Venus}

(The following summary complements the preceding article on Venus by G.E. McGill: not surprisingly, there are some differences in interpretation. Ed.)

At present Venus poses more problems for comparative planetology than it provides answers. It is in many ways similar to the Earth, (e.g. density, bulk composition) but there are also some sharp contrasts. With its intensive gravitational field, Venus is rich in volatiles and has a thick atmosphere consisting mainly of carbon dioxide. Although the Venus' surface has a very high temperature, the content of aqueous vapour in the atmosphere does not exceed 0.004 vol. per cent. Thus, there is the dilemma of whether the venusian material was originally impoverished with water or whether water was lost during the process of its accretion and geological development. The second alternative is more credible, since hydrogen in the venusian clouds is enriched with deuterium.

The most common terrain on Venus consists of "rolling plains" whose hypsometric level is close to the planets' mean radius. Found within their limits are large ring structures, which some workers believe to be ruins of ancient impact craters, indicating that rolling plains are outcrops of ancient terra crust. On the lowland plains, which have generally smoother surfaces, large ring structures are uncommon. The lowland plains appear to represent volcanogenic formations like the lunar maria, although there could also be eolian accumulations in depressions whose origin is obscure.

Dominating the rolling plains are mountain regions, such as Aphrodite Terra, Ishtar Terra and the Beta Regio at $2000 \times$ $2000 \mathrm{~km}$ the largest of the three. These uplifts may be tectonic in character, for radar images, especially of Ishtar, show sets of sinuous parallel lines whose geometry is somewhat similar to the structural folding pattern on terrestrial continents. However, there is not as yet sufficient evidence to confirm this hypothesis. Beta Regio may be compared morphologically with shield volcanoes on Mars and Earth, and there is some indirect evidence that volcanic activity in Beta Regio is fairly young and may even be occurring at present.

\section{Conclusions and Prospects}

The data reviewed here from the the Moon, Mercury and Mars provide evidence that accretion into planets of the preplanetary material was followed by its very early differentiation. Although we do not have a clear idea about the actual mechanisms and energy sources for this differentiation, it also presumably affected the larger planets at an early stage in their development.

A plot of $\mathrm{U}$ against Th for Earth, Moon, Venus, Mars and certain meteorites (Fig. 3A) shows a trend that supports the idea of a common early geological history. K-Th trends for Venus and Earth follow those for chondrites, whereas those for the Moon and for eucrites are distinct and reflect the loss of volatiles by small planetary bodies (Fig. 3B).

Mercury and Mars provide evidence of an early, intensive meteoroidal bombardment which is considered to have affected all solid bodies in the Solar System. The geochemica consequences of this impact appear to have been underestimated, and in order to understand more fully the "laws" of this process it will be neccessary to study in more detail terrestrial impact craters as well as impact features on other planetary bodies.

During later stages of accretion, when planetary bodies grew at a fairly high rate, both projectiles and their target material must have been degassed, and impact metamorphism and melting must also have taken place. On minor planetary bodies this appears to have resulted in a loss of volatiles, whereas on major bodies these must have accumulated to give rise to separate atmospheres and hydrospheres. Thus, the whole geochemical cycle must have commenced at a very early stage in the development of the planets and long before their growth was completed.

Basalt flows are one of the major crustal components of a number of planetary bodies, and are superimposed in some places on more ancient terra material. Basaltic volcanism appears to be associated with the general process of differentiation of planetary interiors, its magnitude being directly related to the dimensions of a planetary body. In contrast, terra material seems to be peculiar in nature to each planetary body, depending upon its dimensions and probably on other unknown factors. Basaltic and terra crustal material may be geochemically related, even though they seem structurally and geologically independent. The resolution of this "contradiction" is one of the long-term objectives of comparative planetology. The unravelling of the postaccretionary evolution of the planets may well come in part from comparative analyses of Earth and Venus, as suggested by the preceding article.

-Prof. V.L. Barsukov is a Director of V.I. Vernadsky Institute of Geochemistry and Analytical Chemistry (Academy of Sciences, Moscow 117334, U.S.S.R.). His scientific interests range from comparative planetology and cosmochemistry to the geochemistry of ore formation and geochemical methods of the earthquake prediction. He is President of IAGC and Chairman of COSPAR's Interdisciplinary Commission on Planets and Small Bodies of the Solar System.

Dr. A.T. Basilevsky is Chief of the Laboratory of Comparative Planetology in the Vernadsky Institute. His scientific interests focus on the synthesis of photogeological and geochemical data from the terrestrial planets.
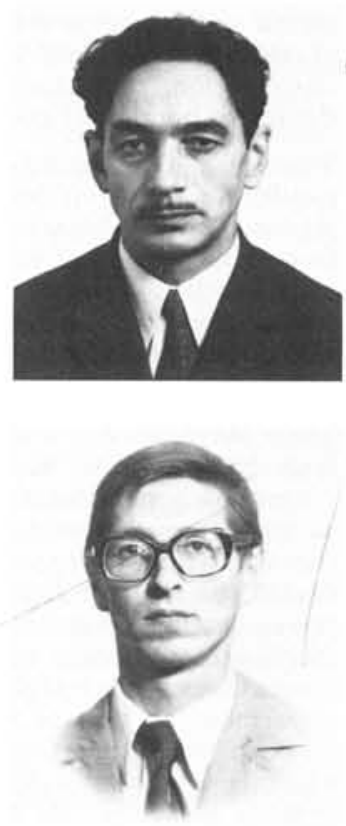